\title{
Commissioning and Testing the 1970's Era LASS Solenoid Magnet in JLab's Hall D
}

\author{
J. Ballard, G. Biallas, G. Brown, D. Butler, T. Carstens, E. Chudakov, J. Creel, H. Egiyan, P. K. Ghoshal (SMIEEE), \\ F. Martin, Y. Qiang, E. Smith, M. Stevens, S. Spiegel, T. Whitlatch and E. Wolin
}

\begin{abstract}
JLab refurbished and reconfigured the LASS, $1.85 \mathrm{~m}$ bore Solenoid and installed it as the principal analysis magnet for nuclear physics in the newly constructed, Hall $D$ at Jefferson Lab. The magnet contains four superconducting coils within an iron yoke. The magnet was built in the early 1970's at Stanford Linear Accelerator Center and used a second time at Los Alamos National Laboratory. The coils were extensively refurbished and individually tested by JLab. A new Cryogenic Distribution Box provides cryogens and their control valving, current distribution bus, and instrumentation pass-through. A repurposed CTI 2800 refrigerator system and new transfer line complete the system. We describe the re-configuration, the process and problems of re-commissioning the magnet and the results of testing the completed magnet.
\end{abstract}

Index Terms-Conducting materials, DC power systems, Magnetic field measurement, Solenoids

\section{INTRODUCTION}

$\mathrm{T}$ HE LASS Solenoid is the principle analysis magnet for the GlueX Experiment in Hall D at the Continuous Electron Beam Accelerator Facility (CEBAF) accelerator at JLab and is shown in Fig. 1. The Hall and recycled superconducting magnet were installed as part of the $12 \mathrm{GeV}$ Upgrade. The magnet was built at Stanford Linear Accelerator in 1971 and reconstituted for the MEGA Experiment at Los Alamos in the 1990s. JLab refurbished, repaired and individually tested the coils and modified the yoke. [1] The magnet consists of four coils in series, contained within an iron yoke $4.80 \mathrm{~m}$ long with a $1.85 \mathrm{~m}$ bore. The solenoid has a peak field on axis of $2.1 \mathrm{~T}$ at $1350 \mathrm{~A}$, with stored energy of $22.8 \mathrm{MJ}$ and an inductance that decreases from $27 \mathrm{H}$ to $25 \mathrm{H}$ with excitation. The coils are wound with open lattice construction and are directly cooled in pool boiling liquid helium. The first generation $\mathrm{NbTi}$ conductor uses a single rectangular strand with 87 filaments sandwiched between and soldered to rectangular copper wire forming a $7.6 \mathrm{~mm} \times 5.3 \mathrm{~mm}$ conductor. The conductor for Coils 1,2 , and 3 have a $\mathrm{Cu}$ to $\mathrm{SC}$ ratio of 20:1 using $0.13 \mathrm{~mm}$ dia. filaments while Coil 4 conductor has a $\mathrm{Cu}$ to SC ratio of 20:1 using 0.18 dia. Filaments. See Table 1 for

XXXXXXXXXXXXXXX Authored by Jefferson Science Associates, LLC under U.S. DOE Contract No. DE-AC05-06OR23177. The U.S. Government retains a non-exclusive, paid-up, irrevocable, worldwide license to publish or reproduce this manuscript for U.S. Government purposes.

J. Ballard, G. Biallas, G. Brown, D. Butler, T. Carstens, E. Chudakov, J. Creel, H. Egiyan, P. K. Ghoshal (SMIEEE), F. Martin, Y. Qiang, E. Smith, M. Stevens, S. Spiegel and T. Whitlatch are with Thomas Jefferson National Accelerator Facility, Newport News, VA, 23606, e-mail: Biallas@jla.org.

E. Wolin is with Health Diagnostic Laboratory Inc., 737 N. 5th Street, Richmond, VA, 23219, e-mail: ewolin@gmail.com. additional construction details.

TABLE I

MAGNET PARAMETERS

\begin{tabular}{|r|r|r|r|}
\hline Coil & Turns & Sub-Coils & Helium Inventory (L) \\
\hline 2 & 928 & 4 & 560 \\
\hline 1 & 1432 & 6 & 1150 \\
\hline 3 & 772 & 4 & 610 \\
\hline 4 & 1476 & 2 & 610 \\
\hline Cryo Box & & & 560 \\
\hline
\end{tabular}

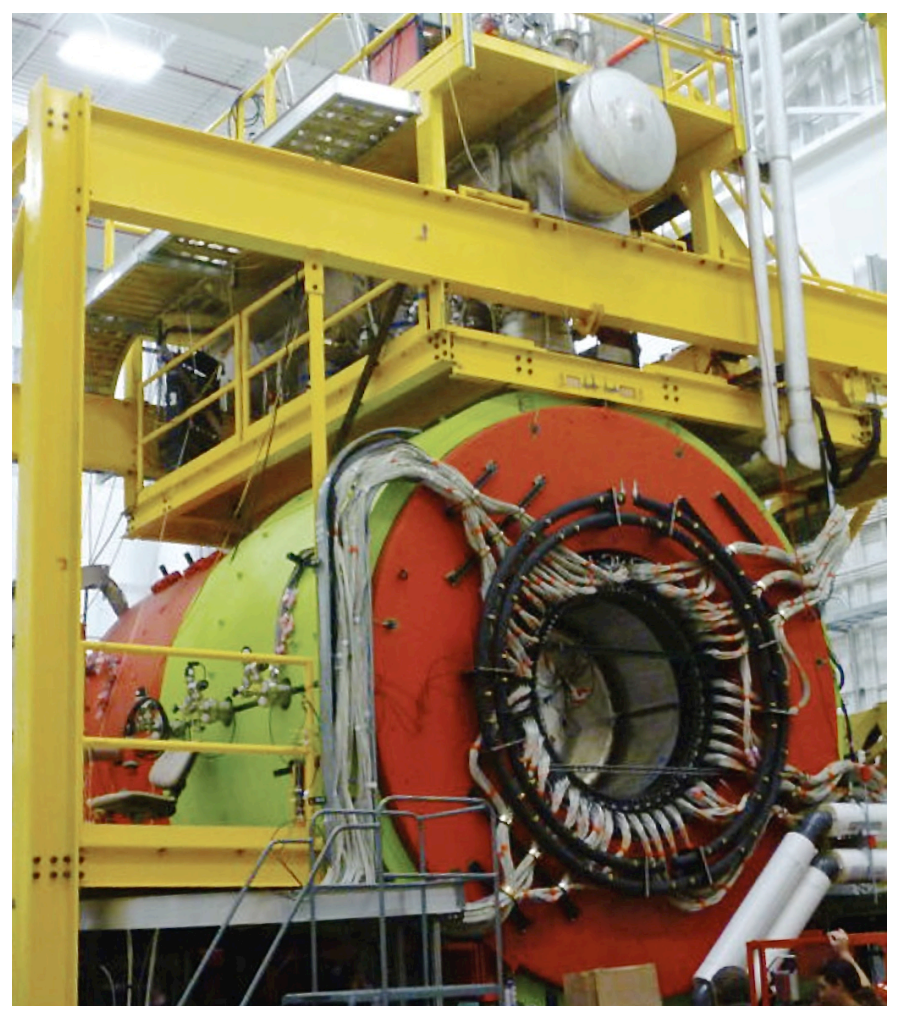

Fig. 1. The Hall D Solenoid

\section{THE JLAB RECONFIGURATION}

\section{A. Magnetics}

Early on, the analysis of the solenoid magnet, we found that machining the upstream yoke pole from $0.41 \mathrm{~m}$ dia. bore to the clear bore of $1.85 \mathrm{~m}$ drastically changed the theoretical axial forces on the upstream coils. The bored out upstream yoke pole doesn't provide a former force balance between yoke and coils, making each of the 24 support columns on Coil 1 subject to a $107 \mathrm{kN}$ compressive load toward the downstream coil at full requested field. The supports are rated at $71 \mathrm{kN}$. 
Our design changes brought this force to the theoretical $22 \mathrm{kN}$ range by interchanging Coils 1 and 2 and adding $76 \mathrm{~mm}$ thick iron irises with a $1.85 \mathrm{~m}$ bore (called baffles) in three locations. These may be seen in Fig. 2.

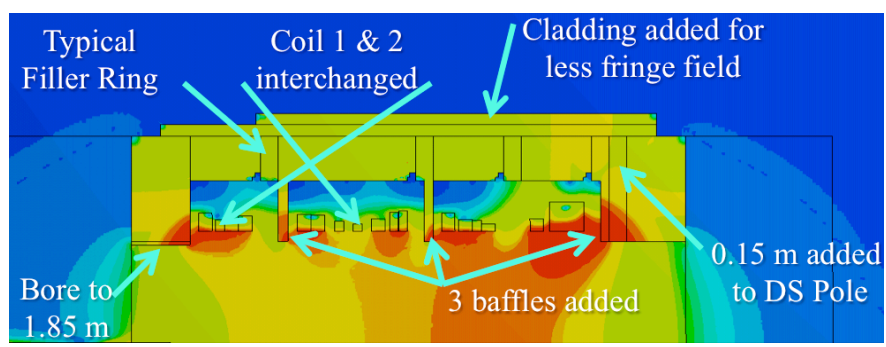

Fig. 2. A half solenoid cross section at $45^{\circ}$ to the vertical with field profiles and yoke changes shown

Magnetic analysis also showed that $5 \mathrm{mT}$ of fringe field, which would interfere with local electronics located around the base of the yoke. We added two courses of $0.15 \mathrm{~m}$ iron cylinders, called cladding, to the outside of the original yoke to stay within this value. Another criterion for fringe field is to bring the field in the Hall to less than $0.5 \mathrm{mT}$ to make the space safe for personnel with electronic medical devices. The cladding necessary for this criterion was excessive. We opted to declare that personnel with electronic medical devices are not allowed in the Hall when the magnet is on.

The original SLAC configuration of the yoke had $0.15 \mathrm{~m}$ gaps at the downstream ends of the four coil yokes. This space housed experimental apparatus and the coil chimneys and vacuum vent pipes to the right side. We filled most of this gap with filler rings. The rings are subject to a $0.30 \mathrm{~m}$ wide interruption at the top for the vertical coil chimneys and a $0.15 \mathrm{~m}$ wide slot on the right side for the original vacuum vent pipes. This asymmetry would cause unbalanced radial forces on the coils and their suspensions. We added balancing interruptions to the filler rings on the bottom and on the left side. The GlueX Experiment requires that the solenoid have a gradient in the field, peaking near the downstream end. Coil 4 has double the number of turns in the same package as Coils 2 and 3 in order to give a boost to the local field. We added an additional $0.15 \mathrm{~m}$ of iron to the downstream yoke pole because analysis showed that field levels in the bore would benefit and the fringe field outside the downstream end would be reduced.

\section{B. Insulation Vacuum Changes}

Because of the history of leaks, we installed vacuum breaks between the four coils and the Distribution Box volume so one leak would not degrade the entire volume. Our individual coil tests proved that the side pump out pipes functioned poorly because of excessive restriction at the entrance to the vacuum volume. We changed the pump out location to the chimneys just above the connection bellows. There, a $\mathrm{LN}_{2}$ shielded break in the superinsulation at the vacuum break provides a good pump out path to the internal vacuum insulation volume of the coils, greatly reducing pump-out times.

A single roots type pump and a flex hose accomplish the initial pump down of the vacuum volumes of the coils or distribution box. The pump evacuates the volumes sequentially into the range of turbo pumps. The pump accesses pump out ports on the right side of the chimneys.

Correspondingly, we use one turbo pump on a common manifold with isolation valves at each coil to continue the pump down to insulating vacuum levels of $\leq 10^{-6}$ Torr. This manifold is on the left side of the chimneys. From this level of vacuum, leak free volumes become self-pumping when liquid helium is in the coils. The difference between the original $3 \mathrm{~m}$ high $\times 3 \mathrm{~m}$ long $\mathrm{x} 1 \mathrm{~m}$ wide SLAC vacuum apparatus and our $0.1 \mathrm{~m}$ dia. Turbo Pump on the common manifold is dramatic.

Additional gas relief of Coil 1, Coil 3 and Coil 4 vacuum volumes, mandated by our pressure system analysis, is provided by flat plate, spring-loaded, O-ring sealed relief plates. No additional machining on the vessels was needed as we incorporated the plates into the existing access covers, used to attach and adjust the radial suspension columns. Relieved gas exhausts into slots machined into the new yoke filler plates and then into the bore. From the bore, venting proceeds to the left and lower gaps in the filler rings mentioned earlier. Coil 2's extra relief is on the chimney with augmentation from a relief on the side vacuum pipe.

Individual vacuum gauging for each volume is resident in the chimney area where a Pirani gauge for crude vacuum and a cold cathode gauge for high vacuum are placed on a manifold with valves at the vacuum volume end and at the pump out end. Faulty gauge heads may be changed out without compromising the main vacuum volumes.

\section{Cryogenics}

The Helium Refrigerator is located in a separate building. The Jefferson Lab Cryo Group took on the responsibility for design and manufacture of the transfer line system from the refrigerator to the Hall and the Distribution Box that supports the Solenoid. The system includes a U-Tube/ bayonet severable interface between the two systems to allow separate debugging and commissioning. The Distribution Box serves the Hall D Solenoid with liquid nitrogen and liquid helium, contains all control valves and all helium circuit instrument feedthroughs, houses the Vapor Cooled Leads and the current distribution bus, which connects the individual coils in series. A G-10 tube based conduit assembly was welded at the bottom of the Helium Vessel by the fabricator. Preplaced pull tapes allowed threading the bus conductor harnesses into the vessel, through the conduits and into their final positions during assembly in the Hall. The bus harnesses consist of two lengths of SSC superconductor braid combined with two lengths of \#2 stranded copper wire. Pull tapes were also preinstalled to aid threading instrument wires to the standpipes with instrument feedthroughs.

\section{Instrumentation and Control}

Our instrumentation and control system uses a Programmable Logic Controller (PLC) for expert control, EPICs for operator control and a National Instruments PXI to provide fast data acquisition and analysis. Our Safety System consists of 15 hard wire or PLC elements that trip the Power Supply to a Slow Dump. Energy is dissipated through the power supply's internal circuitry. Twenty hard wire or PLC 
based elements, including the hard wire Quench Detector (QD), are part of the Fast Dump. The QD compares the voltage of Coils $2 \& 1$ against Coils $3 \& 4$. A fast dump interlock trip opens a Dump Switch that severs contact between the Power Supply and coils. Magnet Current then flows through the current loop formed by a $0.06 \Omega$ Dump Resistor and three diodes in parallel, draining the stored energy with a time constant of 7.2 minutes.

The PLC contains a Sequence Of Events (SOE) monitor feature that the interlock that tripped first. Unfortunately, the SOE never worked for us during all earlier commissioning cycles because of coding and memory glitches. It is running now.

A redundant, independent monitor of current, based on a shunt, is able to open the power supply contactor above a set current. This redundant element compensates for failure modes of the Power Supply's Zero-Flux Current Transducer based current control.

We placed 20-turn pickup coils on the inner, upstream and downstream edges of all four coils. The PXI System, at the start of every ramp, looks for lags in pickup coil voltage signals with respect to current as a sign of turn-to-turn shorts.

\section{E. Installation}

The last coil tested individually was Coil 2 and it revealed an intermittent, ohm level short to ground. After warming up the coil, we burned the short out using capacitive discharge technique. This short was not seen in any later operation.

Coils were left in their stand-up, final configuration while moving them about a quarter mile from the Test Lab to Hall D in order to avoid unnecessary conductor shifts. The Solenoid's yokes and coils were mounted on two piers cast onto the Hall D floor.

\section{1ST COMMISSIONING CYCLE - APRIL/ MAY 2013}

\section{A. Successful Ramp to $1500 \mathrm{~A}$}

In April of 2013, the Solenoid was filled with liquid helium. We checked out all systems and interlocks per a checklist. Immediately on turning on the Power Supply, the hard wire QD tripped. The Power Supply had not been ordered with a tune to the very large inductance of this Solenoid. Transient current pulses at power supply turn on and start of ramp generate enough unbalanced signal to trip the QD. We solved this temporarily by adding a "momentary" switch that jumpered out the hard wire quench detector during transient periods while a person "manned" the switch.

After a nuisance trip at $1360 \mathrm{~A}$, a $6 \Omega$ short to ground appeared in the first pancake of subcoil 3B, nearly the center of the coil sets. This short is consistent with a solder ball bridging the $2 \mathrm{~mm}$ gap between conductor and the bare surface of the coil's support bulkhead. We can run with this one short. A second short creates transformer type loops and can burn out a coil. Detecting a second short is vital. The Power Supply's Ground Fault Detector (GFD) is "blind" to a ground fault at or near its lead's landing point on the coil. This feature allows us to mask our first ground fault but using an indirect method. We landed the GFD lead between two resistors of about $2000 \Omega$ each, with values that match the inductances on either side of the existing ground fault. The opposite leads on the resistors were landed on opposite polarity bus in the power supply. During ramping, the GFD Lead sees zero volts with respect to ground during a ramp. This virtual mask was successful; the GFD did not trip during subsequent ramps.

We ramped to $1500 \mathrm{~A}$, stayed at that current for 20 minutes and then ramped down.

\section{B. The Quench}

The next day, we ramped at a slightly slower rate. At $1460 \mathrm{~A}$, the magnet quenched, venting all helium and contaminating the helium volume because part of the relief was a rupture disk, which we were not prepared to seal. Testing was stopped until a review allowed a go-ahead. Fortunately, our PXI caught the voltage tap voltages shown in Fig. 3.

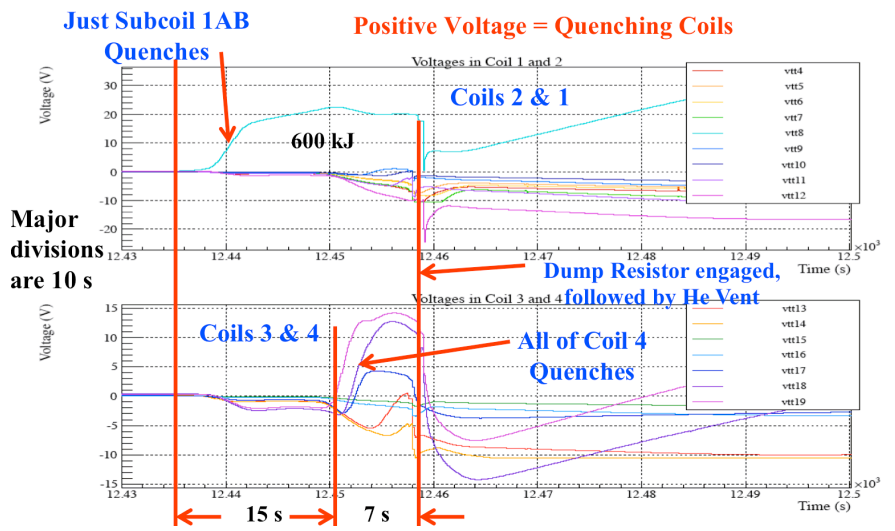

Fig. 3. Voltage tap readings during first $70 \mathrm{~s}$ after quench

\section{Observations on the quench:}

Only Subcoil $1 \mathrm{AB}$ quenched in Coil 1 for the first $22 \mathrm{~s}$ while the adjacent turns of Coil 1, in the main helium chamber, remained superconducting. The Subcoil is housed in an antechamber, isolated from the main chamber with only four $5 \mathrm{~cm} \times 2 \mathrm{~cm}$ openings to the main helium volume. Two of the openings are constricted to $3 \mathrm{~cm} \mathrm{x} 1 \mathrm{~cm}$ by lead insulation.

Helium Bath Pressure was higher at the time of quench $1.35 \mathrm{Atm}=4.56 \mathrm{~K}$, rather than the $1.29 \mathrm{Atm}=4.49 \mathrm{~K}$ when the Solenoid achieved 1500 A.

After $15 \mathrm{~s}$, a second quench engulfed the whole of Coil 4, even though its leads are thermally isolated from Coil 1 . The compressing helium had raised the helium bath pressure to $1.44 \mathrm{Atm}=4.63 \mathrm{~K}$.

Calculations show that the quench was not due to a short and that local heating didn't create a low liquid level in the Subcoil 1AB Antechamber. Problems with conductor damage, leads and splices are also not likely because of earlier running at $1500 \mathrm{~A}$.

Further analysis of raw, un-averaged voltage tap data showed small voltage spikes on voltage taps of other coils during ramping, perhaps indicating small movements. Unfortunately, Coil 1AB's voltage tap signal was too noisy to discern a similar (or bigger) voltage spike at the time of 
quench. We believe heat from a large conductor motion is not ruled out as a cause of the quench.

Our analysis now shows that the conductor filament size is too large to inhibit flux jumps. The conductor specification didn't benefit from the theory of the use of small filaments because the theory was developed about the same time.

A sample of this first generation conductor, of unknown reel number, was tested at the University of Twente to measure short sample current vs. temperature at two field levels. The slope is $-1000 \mathrm{~A} / \mathrm{K}$ compared to $-414 \mathrm{~A} / \mathrm{K}$ for specification conductor. Bath temperature has a large affect.

Our path forward was determined by a review where Leigh Harwood, the reviewer representing the $12 \mathrm{GeV}$ Project presented Table 1, summarizing our views and establishing policy:

TABLE I

EVALUATIONS OF THE CAUSE OF THE QUENCH

\begin{tabular}{|l|c|}
\hline Fault & Evaluation \\
\hline 1. Short Developed & No \\
\hline 2. Problems at splices & Very \\
3. Conductor Damaged & Unlikely \\
\hline 4. Gas bubble exposed Conductor & Unlikely \\
\hline 5. Flux jump or & Possible \\
6. Conductor motion & \\
\hline
\end{tabular}

No "Smoking Gun"

- We identified two possible explanations

- Solenoid will operate at $\leq 1350 \mathrm{~A}$

\section{2ND COMMISSIONING CYCLE - JULY/ AUGUST 2013}

\section{A. Successful Limited Ramp}

We used the period before the next cycle to make our Power Supply voltage spike free. We installed circuitry to tune for our $\sim 26 \mathrm{H}$ inductor and installed a programmed ramp feature in the firmware that minimizes spikes on current change. We also installed a Temperature/Current Margin Monitoring System. The PLC issues warning when the bath temperature is high enough to encroach on a $135 \mathrm{~A}$ margin from possible quench and the PLC trips a slow dump when a $110 \mathrm{~A}$ margin is breached.

The goal of our second testing cycle was to (1) prove the Solenoid was still intact, (2) that it would be stable at 1300A after a hysteresis cycle to $1350 \mathrm{~A}$ and to (3) magnetically map the Solenoid.

The Cryo Group filled the solenoid with liquid helium - but with difficulty. The CTI 2800 Refrigerator started to plug with carbon dust abraded from the carbon beds at the very heart of its heat exchangers. The refrigerator capacity decreased drastically and blow-downs to vent the carbon accumulations were only partially successful. During blow downs, no liquid helium supply was available to the Solenoid. The Cryo Group augmented the refrigerator during these cycles in operation using $1000 \mathrm{~L}$ Dewars of LHe obtained from other sources on site.

The programmed ramp and tuning additions to the power supply worked without any problems. No voltage spikes were seen. Vexingly, fast dumps at around 1000 A plagued us. Unfortunately our attempts at fixing our SOE System failed again and we were blind to the cause of the trips. After several shifts of failed ramps, we found the cause of the trips. The Vapor Cooled Lead (VCL) helium flow controllers/meters indicated "no flow" for a fraction of a second and initiated the fast dump via their hard wire interlock. We jumpered the controllers out of the interlock system and set the helium flow to that required for maximum current by adjusting and locking the controller's isolating valves. The control system has several other methods of safeguarding the Solenoid from VCL failures, so we remained protected. The cause of this anomalous flow reading remains unexplained.

The solenoid easily ramped to $1360 \mathrm{~A}$, as the top current of a small hysteresis cycle, and was brought back to $1350 \mathrm{~A}$.

\section{B. Field Mapping}

We measured the Solenoid at its central bore at 1350 A over about 3 hours. We then ramped down to $1300 \mathrm{~A}$ for mapping at characteristic positions throughout the bore over 18 hours. About 12 hours into the 1300A mapping, the field was ramped down to accommodate a 1000 L Dewar "fill". Fills boost bath temperatures above the current margin limits for $1300 \mathrm{~A}$. After liquid helium level was recovered, we ramped back up to $1300 \mathrm{~A}$ for more measurements.

The mapping system was based on a plastic slug containing a multi axis Hall Probe that was pushed in, by hand, to predetermined axial positions in an aluminum tube that was straight to within fractions of a $\mathrm{mm}$ and positioned in the bore to a similar tolerance. A laser based distance meter of similar resolution and an accurate electronic level read out the exact position of the slug and its orientation. The aluminum tube was placed to the follow-on, representative positions within the bore to sub $\mathrm{mm}$ accuracies by simple fixturing. The limited mapping obtained the data necessary to generate the field map necessary for Physics.

\section{3RD COMMISSIONING CYCLE - JULY/ AUGUST 2014}

The Cryo Group completely refurbished the refrigerator, ridding it of any carbon beds and finding that it has the capacity to cool down the Solenoid and fill it with liquid. To save time, they did augment the latest fill with two $1000 \mathrm{~L}$ Dewars. The Helium Bath's equilibrium pressure is $1.23 \mathrm{Atm}$ $=4.45 \mathrm{~K}, 0.05 \mathrm{~K}$ lower than the temperature during the Solenoid's 1500 A cycle. The magnet is now being checked against our checklist before a planned extended run at $1100 \mathrm{~A}$.

\section{CONCLUSIONS}

The Solenoid is robust. It is proven to withstand a quench and fast ramp downs without damage. We can run with the higher resistance, intermittent ground fault. Recent tests show $I_{c}$ is a strong function of Bath Temperature for this $1^{\text {st }}$ generation conductor. The newly refurbished Refrigerator with its lower bath pressure may allow us to run at currents closer to our original goal of $1500 \mathrm{~A}$.

\section{ACKNOWLEDGMENT}

This work was supported in part by the U.S. Department of Energy under U.S. DOE Contract No. DE-AC05-06OR23177. 


\section{REFERENCES}

[1] J. Ballard, G. Biallas, P Brindza, T. Carstens, J. Creel, H. Egiyan, F. Martin, Y. Qiang, S. Spiegel, M. Stevens, M. Wissmann and E. Wolin, "Refurbishment and testing of the 1970's era LASS solenoid coils for JLab's Hall D" AIP Conf. Proc. 1434, 861 (2012); 13-17 June 2011 Spokane, Washington, USA

[2] N. Laverdure, J. Creel, K. Dixon, V. Ganni, F. Martin, R. Norton, S. Radovic, "The Hall D Solenoid Helium Refrigeration System at JLab," AIP Conference Proceedings; Jan2014, Vol. 1573, p329.

[3] L. Harwood, G. Biallas, E. Chudakov, R. Fair, P. Ghoshal, R. Ghoshal, E. Salpietro Y. Qiang, G. Young, "Investigation of Hall D Solenoid Quench," Internal JLab Document, Sept 27, 2013 